\title{
固体 $\mathrm{C}_{60}$ 电阻的压力效应
}

鲍忠兴 顾惠成 王积方 陈 红

(中国科学院物理研究所, 北京 100080)

李玉良姚幼新 朱道本

(中国科学院化学研究所, 北京 100080)

\section{关链词固体 $C_{60} 、$ 压力效应、相变}

固体 $\mathrm{C}_{60}$ 的发现引起了人们很大的兴趣, 它的研究已成为一个相当活跃的新领域. 单个的 $\mathrm{C}_{60}$ 分子具有稳定的球形笼状结构, 而固体 $\mathrm{C}_{60}$ 是靠范德瓦尔斯力结合的, 是一种分子固体, 其 结构为面心立方. 固体 $\mathrm{C}_{60}$ 的能隙约为 $1.5 \mathrm{eV}^{[1]}$, 相当于本征半导体. 掺人碱金属后呈现超导 电性, 其超导转变温度可达 $33 \mathrm{~K}^{[2]}$. 近年来, Duclos 等 ${ }^{[3]}$ 和 Núñez-Regueiro 等 ${ }^{[4]}$ 已在高压下 对固体 $\mathrm{C}_{60}$ 的晶体结构和电学性质等进行了研究, 他们都发现固体 $\mathrm{C}_{60}$ 在 $15 \mathrm{GPa}$ 或 $20 \mathrm{GPa}$ 以 上的压力下有一个相变, 而且在 Núñez-Regueiro 等可, 他们还采用快速和非静水压压缩的方 法在 20-25 $\pm 5 \mathrm{GPa}$ 的压力下观测到了固体 $\mathrm{C}_{60}$ 转变成金刚石. 本文报道了我们研究固体 $\mathrm{C}_{60}$ 在高压下电阻变化所得到的实验结果, 并对结果进行了初步讨论.

\section{1 实 验}

本工作在金刚石压砧装置上采用电阻方法测量了固体 $\mathrm{C}_{60}$ 在室温下, $27 \mathrm{GPa}$ 内电阻与压 力的关系. 实验装置和测量方法与我们过去采用的相同 ${ }^{[6]}$. 金刚石压砧顶面为正十六边形, 其 直径为 $0.5 \mathrm{~mm}$. 两钿片电极间的距离为 $0.03-0.05 \mathrm{~mm}$. 样品为多晶粉末, 纯度为 $99.99 \%$.

\section{2 结果与讨论}

实验中, 测量了三个样品的电阻一压力 $(R-P)$ 关系. 测量前固体 $\mathrm{C}_{60}$ 样品的起始电阻值 均大于 $1000 \mathrm{M} \Omega$ 。 由于实验中采用的仪器为电子管电压表, 它的电阻测量极限值是 $1000 \mathrm{M} \Omega$, 因此测量中在 $8 \mathrm{GPa}$ 以前没有观测到样品电阻的变化. 样品的电阻随着压力增加较快地减 小, 在 $22-23 \mathrm{GPa}$ 左右, 电阻突然增加, 而且增加得很缓慢. 为了仔细观测这个现象, 工攺沪 对三个样品分别进行了三次不同的实验: 当电阻突然增加时, 使压力保持不变, 在这个压力下 分别停留 $21 、 136$ 和 $350 \mathrm{~min}$, 此时电阻的相对增加量 $\Delta R / R_{1}\left(R_{2}-R_{1} / R_{1}, R_{1}\right.$ 为在此压力下 停留开始时, 即电阻突然开始增加时的电阻值, $R_{2}$ 为在此压力下停留结束时的电阻值)分别为 $35 \% 、 61 \%$ 和 $66 \%$. 在这以后, 随着压力的增加, 电阻又逐渐减小. 实验中发现: 在第一、二 个样品中, 从电阻突然增加时开始, 虽然在该压力下分别停留了 $21 、 136 \mathrm{~min}$, 但电阻仍在很缓 慢地增加; 而在第三个样品的测量中, 从电阻突然增加时开始, 在此压力下, 经过 $330 \mathrm{~min}$ 以后, 电阻已不再增加了 图 1 表示第一个样品的实验结果, 可以看到, 在降压的 $R-P$ 曲线上没有 观测到电阻突然减小的变化. 我们认为, 实验中所观测到的电阻突然增加是一个相变, 由半 


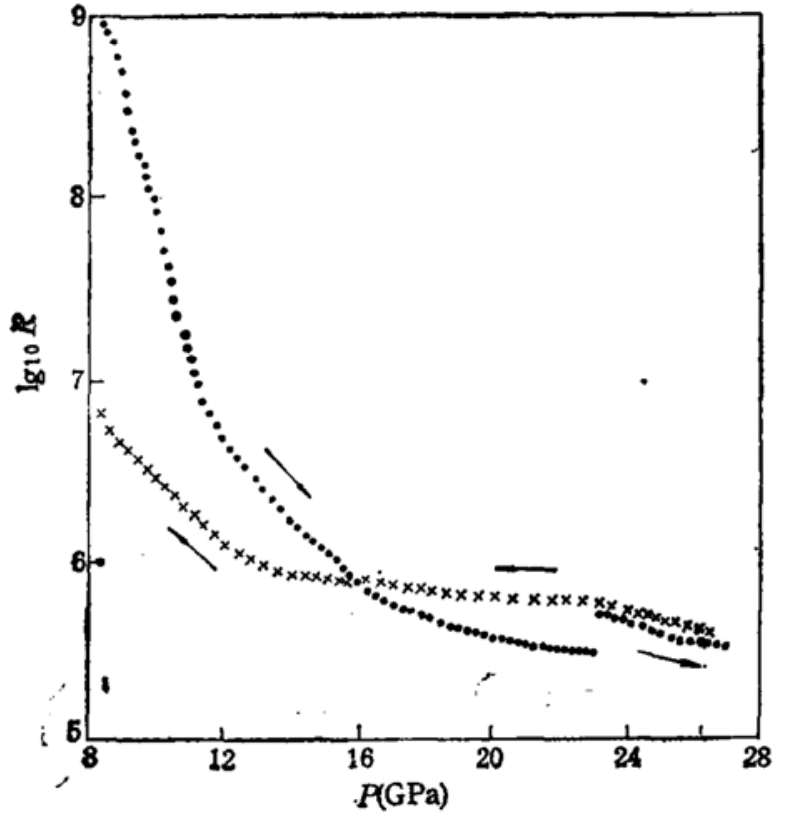

图 1 固体 $\mathrm{C}_{60}$ 的 $R-P$ 关系

导㑀相变成绝缘相. 这个结果与 NúñezRegueiro 等 ${ }^{[4]}$ 的实验结果是一致的. 他们在 聚晶金刚石压砧装置上采用电阻测量方法观 测到了固体 $\mathrm{C}_{60}$ 在 $15 \mathrm{GPa}$ 或 $20 \mathrm{GPa}$ 以上的 压力下有一个相变. 本实验中所观测到的这 个绝缘相就是 Duclos 等 ${ }^{[3]}$ 通过高压 $\mathrm{X}$ 光衍 射测量所观测到的低对称相, 而且在不同的 $\mathrm{C}_{60}$ 原子簇之间可能已形成了具有 $s p^{3}$ 杂化轨 道的共价键.

我们还对卸压后的样品进行了 $\mathrm{X}$ 光衍射 分析, 发现卸压后的样品已变成非晶态. 这 说明固体 $\mathrm{C}_{60}$ 样品在 $27 \mathrm{GPa}$ (测量中所达到 的最高压力）下已由相变后的低对称相变成 了非晶态 (从 $R-P$ 曲线上没有观测到电阻的 明显变化), 而且这个非晶态在卸压后被保留 下来了. 这个结果与 Núñez-Regueiro 等凸 所得到的实验结果也是一致的. 他们也得到了固体 $\mathrm{C}_{60}$ 在高压下变成非晶态的实验结果. 这 个非晶态有可能是具有 $s p^{3}$ 杂化轨道的非晶碳.

从我们的实验结果可以看到, 固体 $\mathrm{C}_{60}$ 在相变时, 电阻的突然增加是非常缓慢的, 这个相 变是一个很缓慢的过程, 需要 5 个半小时左右的时间才能完成. 我们认为, 这个绝缘相在形成 的过程中, 压力一方面促使不同的 $\mathrm{C}_{60}$ 原子簇之间由原来的范德瓦尔斯键可能转变成具有 $s p^{3}$ 杂化轨道的共价键, 而另一方面也在促使每个 $\mathrm{C}_{60}$ 分子里的 $s p^{2}$ 杂化轨道转变成 $s p^{3}$ 杂化轨道, 由于每个 $\mathrm{C}_{60}$ 分子是一个成键十分紧密的、难于压缩的球形结构, 因此这个转变的过程是一个 很缓慢的过程,需要较长的时间才能完成. 另外,在不同的工作和同一工作的不同的实验中, 所观测到的固体 $\mathrm{C}_{60}$ 的相变压力不同,这可能是由于静水压的程度不同所引起的.

致谢 工作中得到了李荫远先生的指导和支持,同时还得到了陈良辰、车荣钲和王莉君等 同志的帮助,并与他们进行了有益的讨论.

\section{考文献}

[1] Saito, S., Oshiyama, A., Phys. Rev. Lett., 1991, 66(22): 2637-2640.

[ 2 ] Fleming, R. M., Ramirez, A. P., Rosseinsky, M. J. et al., Nature, 1991, 352(6338): 787-788.

[ 3 ] Duclos, S. J., Brister, K., Haddon R. C. et al., Nature, 1991, 351(6325): 380-382.

[ [4] Núnez-Regueiro, M., Monceau, P., Rass3t, A. et al., Nature, 1991, 354(6551): 289-291.

[ 5 ] Núñez-Regueiro, M., Monceau, P., Hodeau, J. L., Nature, 1992, 355(6357): 237-239.

[6]䱒忠兴、张芝婷、俞汀南,科学通报, 1984,29(14): 846-849.

[ 7 ] Kriza, G., Ameline, J. C, Jerome, D. et al., J. Phys. I (France), 1991, 1(10): 1361-1364. 